

\title{
الحوار المخملي كآلية للتواصل أثناء الإضرابات الطلابية
}

\section{Velvet dialogue as a mechanism for communication during student strikes}

تاريخ الاستلام : 2019/08/23 ؛ تاريخ القبول : 2019/09/17



\section{Abstract}

Velvet dialogue is considered an intermediate strategy to reduce many social, professional and educational problems by employing the actual linguistic practice in social situations and possessing polite and simple oral communication skills that achieve the tension between the actors in any institution.

This dialogue has become necessary especially among the actors in the university institution, which is now living in the face of conflicts and periodic differences between the university administration and university students, which negatively affected the educational process in university life, when it is necessary today to rely on a dialogue between these, based on A culture of respect and recognition of the other and the recognition of the right and review matters, considering that this method is the solution to the problem and put an end to these repeated student strikes that do not serve the Algerian Universit

Keywords: dialogue, velvet dialogue, communication, students, student strikes.

\section{Résumé}

Le dialogue de velours est considéré comme une stratégie intermédiaire pour réduire de nombreux problèmes sociaux, professionnels et éducatifs en utilisant la pratique linguistique réelle dans des situations sociales et en possédant des compétences de communication orale polies et simples qui créent la tension entre les acteurs de toute institution.

Ce dialogue est devenu nécessaire, en particulier parmi les acteurs de l'institution universitaire, qui vit maintenant face à des conflits et à des différences périodiques entre l'administration universitaire et les étudiants universitaires, qui ont affecté négativement le processus éducatif de la vie universitaire, alors qu'il est aujourd'hui nécessaire de: s'appuyer sur un dialogue entre ceux-ci, fondé sur une culture de respect et de reconnaissance de l'autre et sur la reconnaissance du droit et le contrôle, en considérant que cette méthode est la solution au problème et de mettre un terme à ces grèves répétées des étudiants qui ne le sont pas servir l'université algérienne.

Mots clés: dialogue, dialogue de velours, communication, étudiants, grèves des étudiants.

* Corresponding author, e-mail: bouab.redouane@yahoo.fr 


\section{أولا : مدخل بصيغة...إشكال:}

إن المجتمع هو أكبر وحدة للتحليل السوسيولوجي ، يضع عدة وحدات اجتماعية







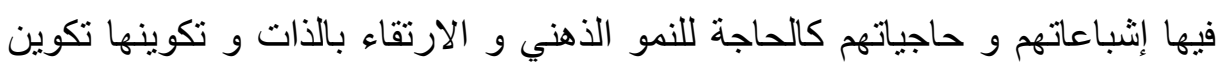

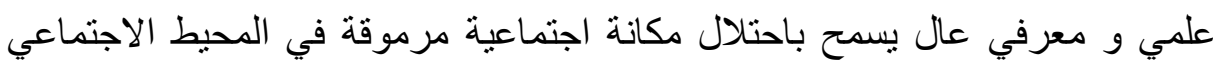





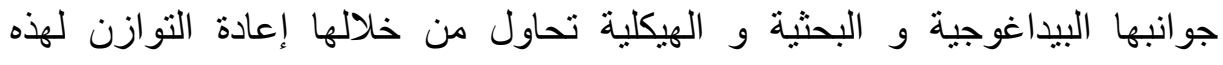








ورفع كل هذه الإصلاحات و الإنجازات في قطاع التعليم الجامعي إلا أنه كانت







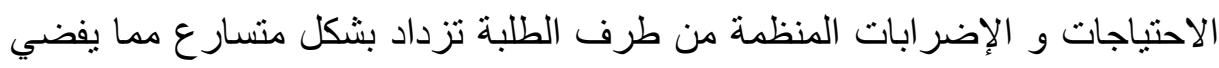

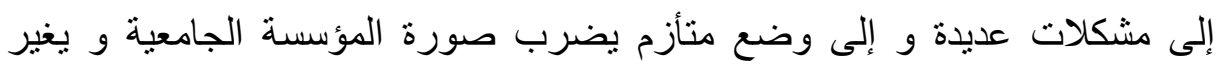
بعملية تحقيق الأهداف التعليمية المنشودة. إن طبيعة هذه الإضرابات و المشكلات الحاصلة تعود في الغالب إلى غياب ثقافة
















وجودة في العملية التعليمية ، وفتح قنوات الاتصال مع ممثلي الطلبة و منظماتهاتهم












يصبح السمو و الرقة و اللطف و المراعاة و التفاعل الإيجابي شيئا من مكونات الذات و ملامحها العامة (1) ومنه يجب أن يكون هذه الحوار مباشرا وجها لوجه أساسه

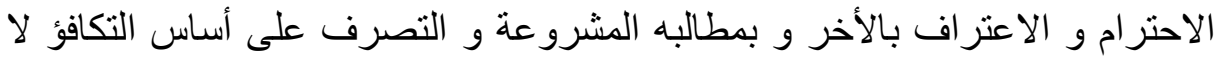





فالحوار الجيد في المؤسسة الجامعية حول مشاكل العمل العمل و و حول المشاكل



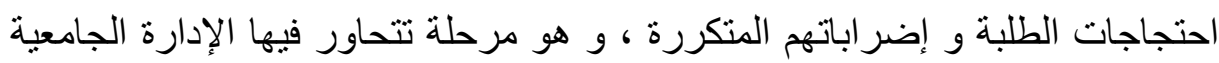



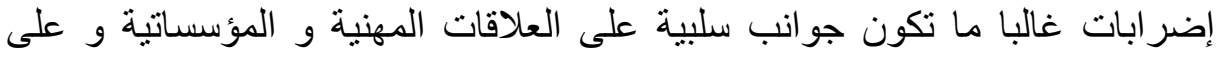






محورية الموضوع الذي أرق العديد من أصحاب القرار الجامعي، و كان مصدر قلق بلق لإن



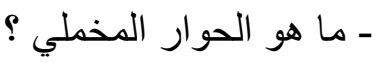

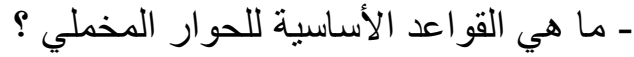
ـ ما هي الفو ائد المترتبة عن العملية الحوارية أثناء الإضر ابات الطئة الطلابية ؟ ـ ما هي آليات الحوار المخملي المساعدة على الحد من الاحتياجات عند الطلبة ؟ ثانيا : أهداف المقال : التات







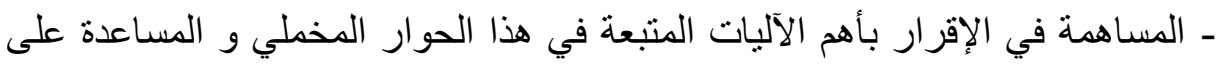
الحد من الاحتياجات عند الطلبة.

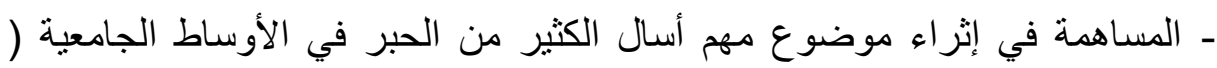

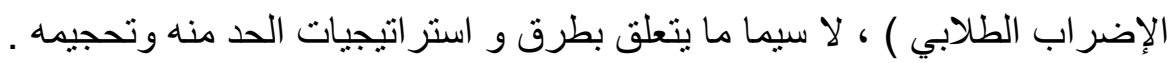

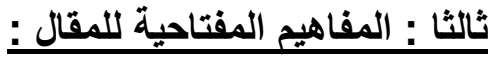
1- الحوار : يعرف لغة : بمعنى المحاورة و مراجعة الكلام بين اثنين و قد عرفها ابن

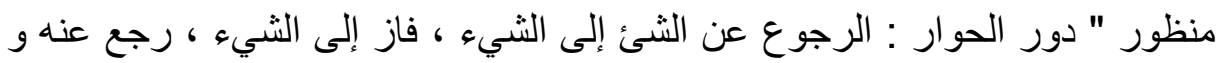





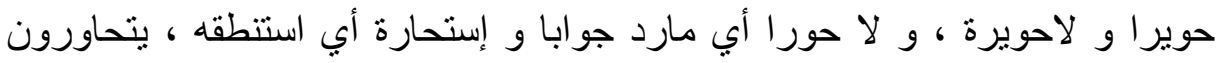





عنه بخبر (3) و الحوار اصطلاحا : حديث يدور بين اثثين أو أكثر يقوم على مناقثة متبادلة بين 


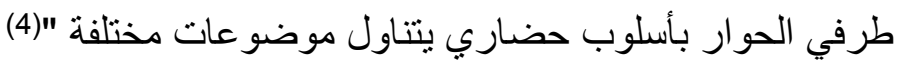

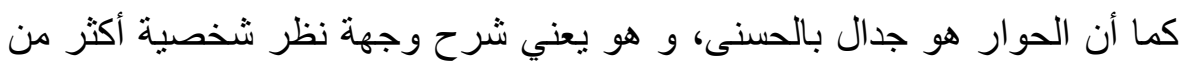


فالحوار هو ممارسة وسلوك يومي و حياتي يتم بمناقثة الكلام بين الأشخاص بهدوء و احتر ام ودون تعصب للر أي ، و يهدف من خلالله الفرد إلى التو اصل مع الته البيئة

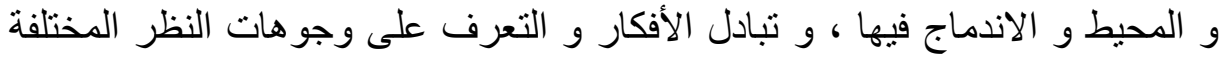

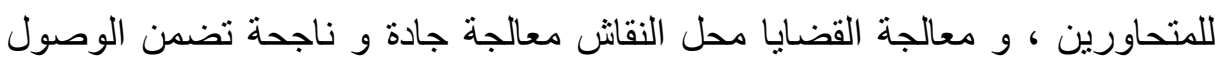
إلى حقائق وحلول . 2/ المخملي : تعريف لغة من الفعل خمل منسوب إلى ( المخمل )فعل يخمل خمولا و






و عليه فالصوت المخملي هو الصوت المنخفض، و خفض الرف الرجل صوته لهفه خملا أي خفضه و أخفاه و لم يرفعه، و بذلك فإن الحوار المخملي هو الحوار الذي تكون فيه


3/ الحوار المخملي: يعتبر هذا النوع من الحوار من الحوارات الغير مستعمل بين




الثفهي و اللغوي و الاجتماعي . التيني و الحوار المخملي " مصطلح جديد أريد منه ذلك النوع من الحوار القائم على الأناقة و الأمي


الموروث الذي ينطلق فيه الناس على سجيته دون اهتمام بالتفاصيل ودون التهون اهنمام





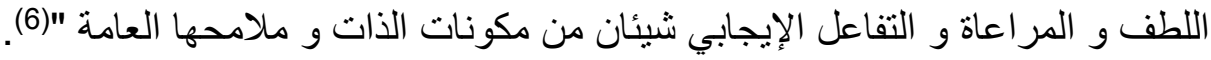

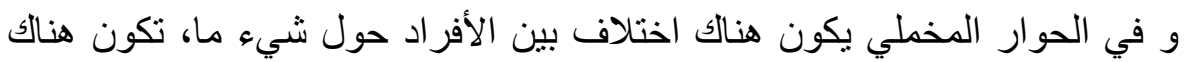

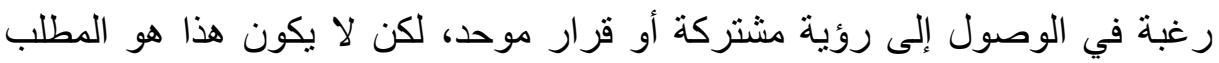

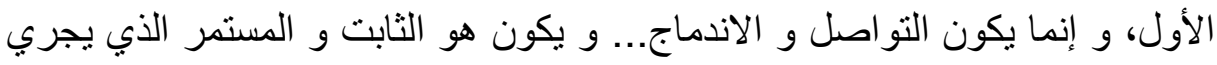

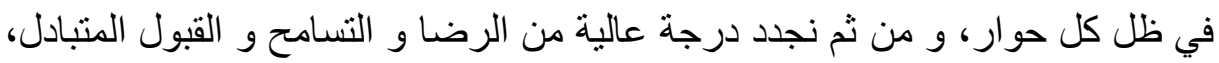
و كأنه ليس هناك خلاف أو نز اعل في في فئل فئلة من المسائل....

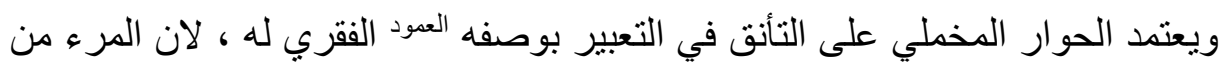

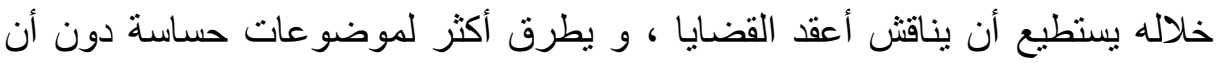

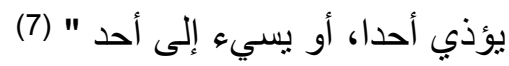
و إجمالا فالحوار المخملي هو الحوار الهادئ المهذب و اللطيف و الذي نستعمل

فيه الخطاب السهل و الناعم، حيث يصل بين المحاورين بطريقة سهلة و سلسة. 4/ التواصل : إن التواصل هو تقنية إجرائية أساسية في فهم التفاعلات البشرية حيث الثين 
أصبح التو اصل علما قائما بذاته ، له مقوماته الخاصة و أساليب و أثنكاله المحدودة له

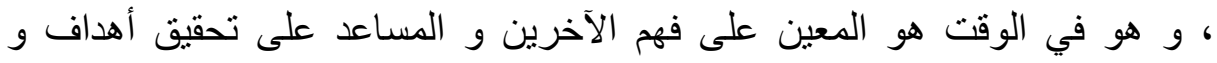

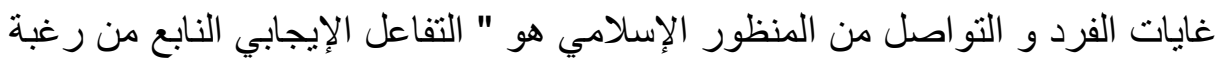
صادقة في خلق التفاهم من الآخرين(8).

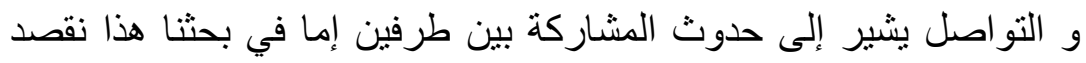

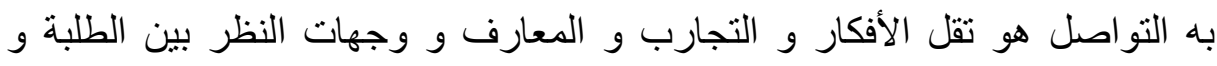
الإدارة كفاعلين في المنظومة الجامعية بغية التفاهم و التفاعل الإيجابي وحل المشكل الشكلات

5/ الطالب الجامعي: يعتبر الطالب من أهم العناصر المساهمة في إنجاح العملية التعليمية في الجامعة و هو الفرد الذي أصبح بستثمر فيه في هذه المرحلة التعليمية.

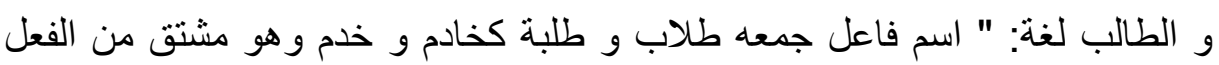
طلب أي رغب وقيل محبة وصول الثيء على وجه يقتضي السعي في التحصيل لو لالا مانع الاستحالة و البعد كما في التمني "(9).

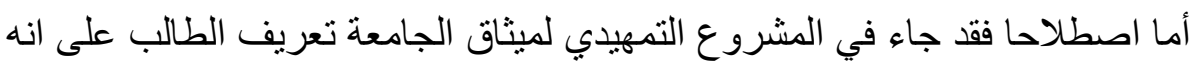
" يعطي اسم طالب على كل شخص يسجل بصفة منتظمة في مؤسسة من مؤسسات التعليم العالي، و ذلك من أجل مواصلة تكوين للحصول على شهادة "(10) .








و إجرائيا فإن الطالب هو ذلك الثخص الذي سمحت له مؤهلاته بالانتقال من المرحلة

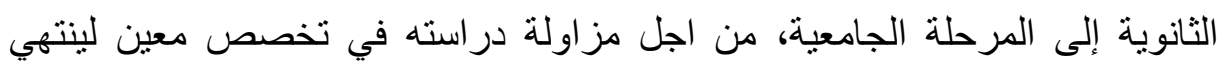
بالحصول على شهادة ممنوحة من طرف المؤسة الجسة الجامعية.








التعليمية في ذلك الموسم الجامعي . التعائ. رابعا : أهمية الحوار المخملى أثناء التوترات التوات الطلابية :









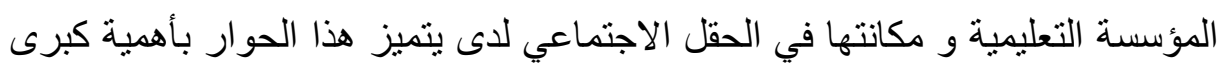
و في عديد من الجوانب منها : الجاتب المعرفي : فعن طريق الحوار تكون هناك تنمية معرفية و فكرية للمتحاورين ،




عنها مناقثنة بناءة و موضوعية ، و يزيد تحصيلهم المعرفي من خلال الحصول على ملى

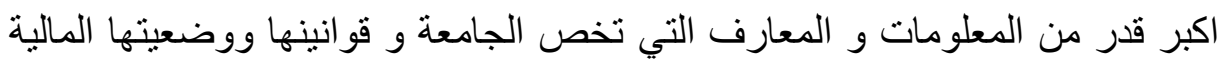
، البيداغوجية ،الهيكلية مما يسمح بإيجاد أرضية مناسبة و عملية الانطلاق في العملية التفاوضية . التئ.

الجانب النفسى الاجتماعى : إن العملية الحوارية المخملية تدعم الثقة في نفسية

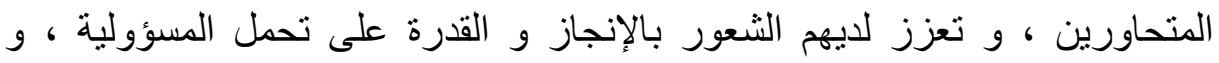

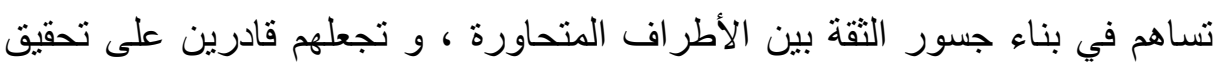

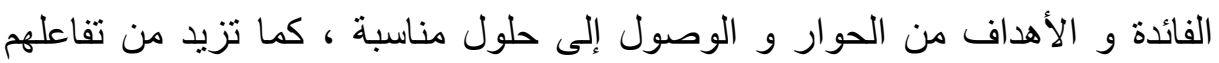

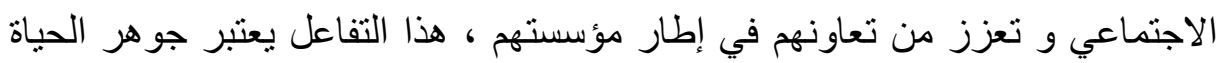

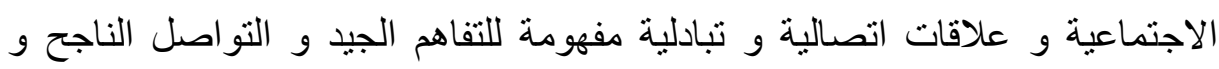

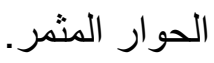
الجاتب التربوي : في هذا الجانب يتم تعديل بعض السلوكيات الغير مقبولة اجتماعيا

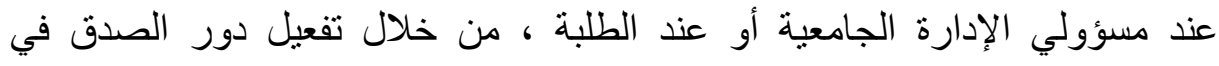

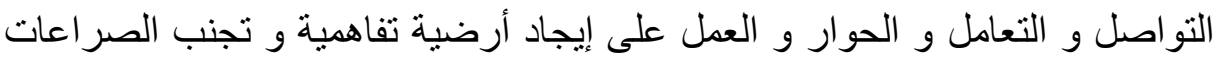

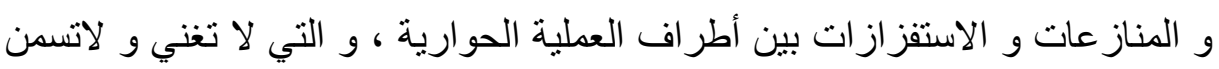

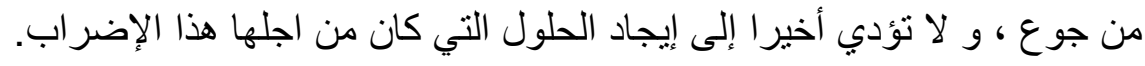

\section{خامسا : القواعد الأساسية للحوار المخملى الناجح :}

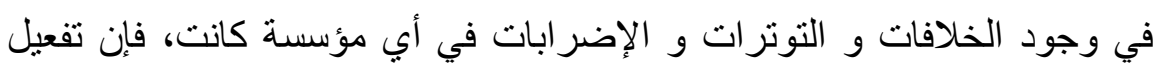

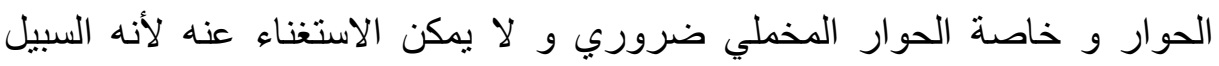

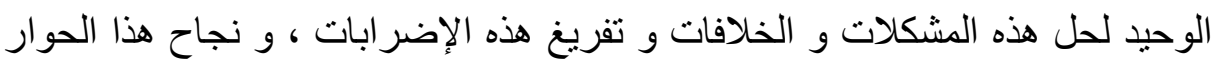
يكون من خلال قو اعد أساسية و امتلاك مهار ات معينة يمكن حصر هات لفهات فيما يلي (12).

ــ الإنصات : هو من المهار ات المهمة في أبي جلسة حوار بين أكثر من متحاور ويؤثر

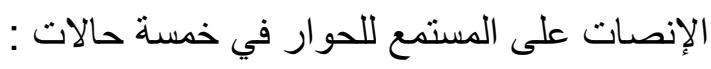

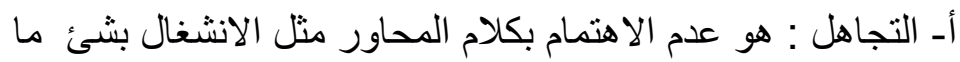

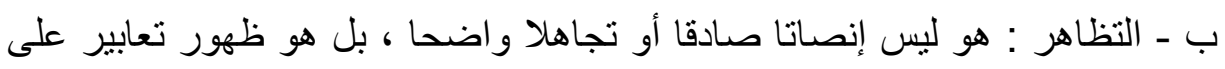



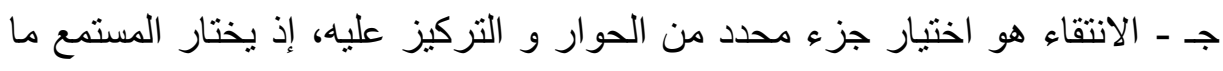

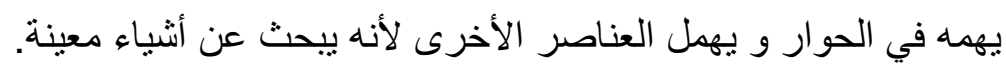

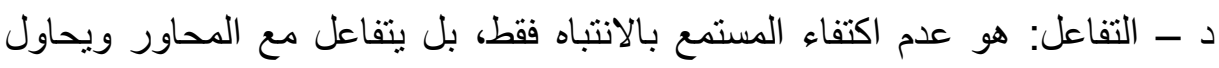
مناقشته و التعرف على ر أيه وخصوصا في حال وجود اختلاف في الآراء.

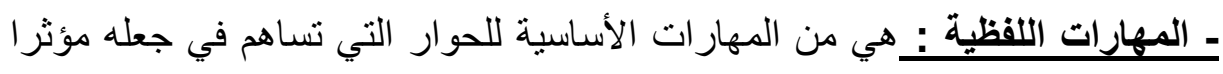
على المستمعين و من أهم هذه المهار ات اتهي من المهارات :

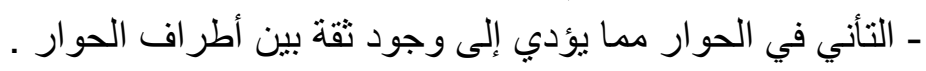
ـ التتويع في نبرة الصوت في أثناء التكلم 
- تغيير طبيعة سرعة على نحو متدرج ، و التوقف قبل وبعد الحديث على الأمور المهمة . ـ تجنب رفع الصوت ( الصر اخ) لأن المستمعين ينجذبون إلى المحاور المخملي

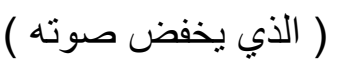

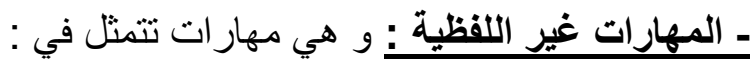

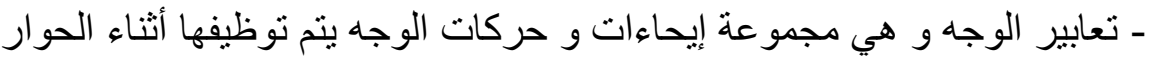

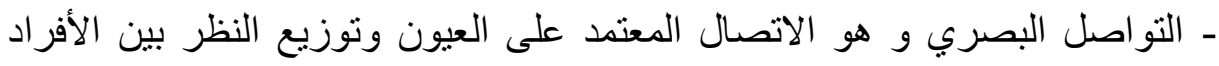

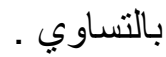
- حركة اليدين و هي مهارة تشكل انطباعا إيجابيا حول شخصية المحاور .

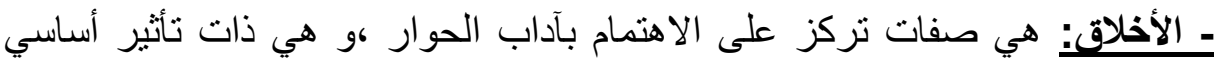
على سير النقاش و تضمن استمرارية بطريقة صحيحة ، لإى يتطلب تجنب الإب الأمور

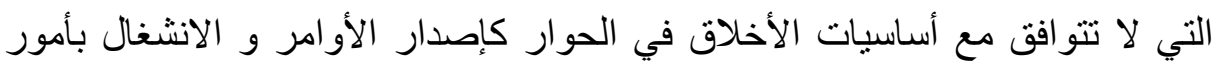

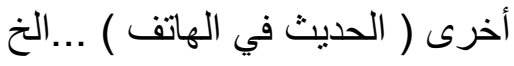
كما يتركز الحوار الناجح عن البعض على مرئ مرتكز ات يمكن حصر ها فيما يلي :

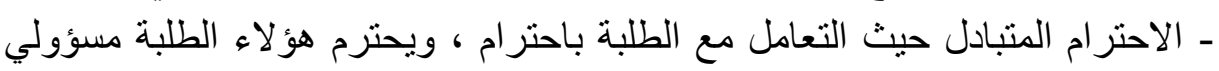

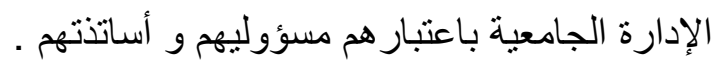

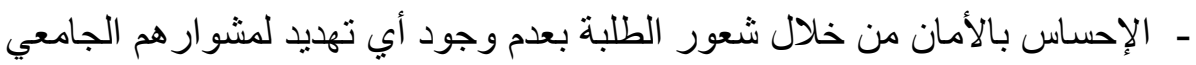

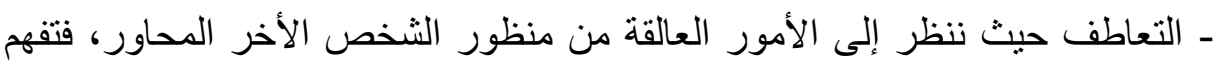

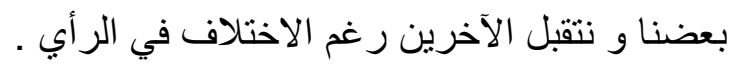

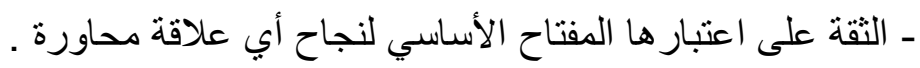

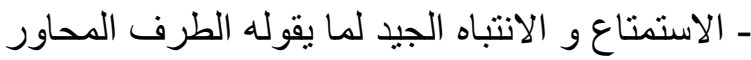

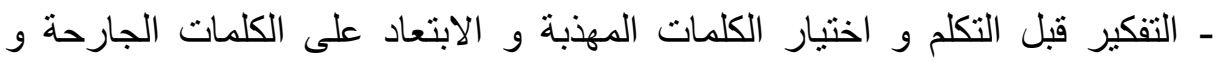

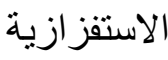

- عدم المقاطعة ورفع الأيدي أثناء العملية الحوارية

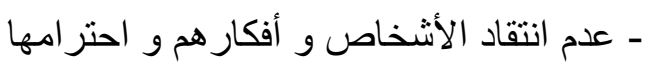

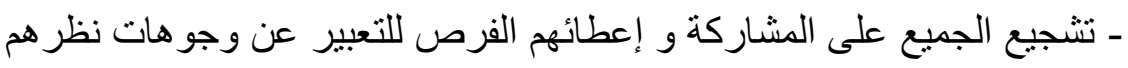
- حسن الخلق و الابتعاد عن الغضب الذي يؤدي إلى مالا يحمد عقباه

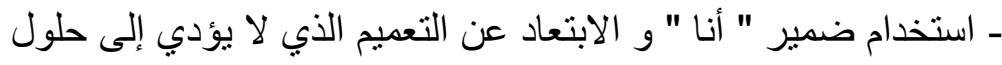

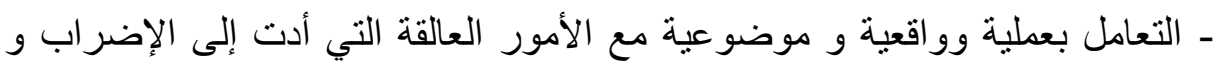

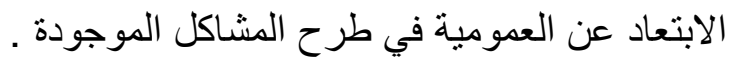

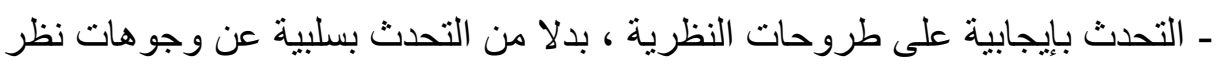
الآخرين ـ الاعتر اف بالنقائص الموجودة و الدعوة للتعاون علو حلهاو إثر الك الطرف الأخر .

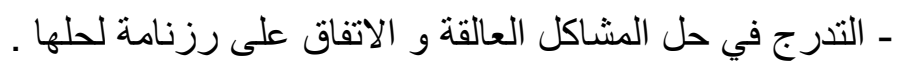

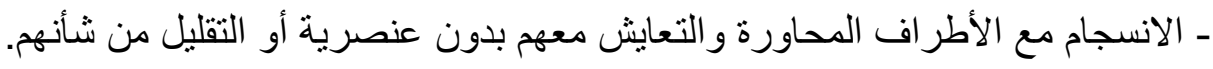
ـ الثفافية و الصدق في السعي لإيجاد الحلول. - فسح المجال للتعبير عن وجهات نظر المحاورين و عدم الاستهزاء بها ولو كانت 
غير موضو عية و مثالية التحقيق.

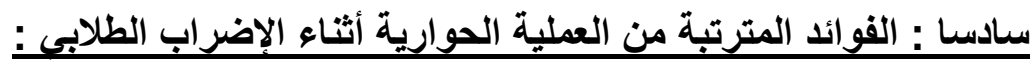

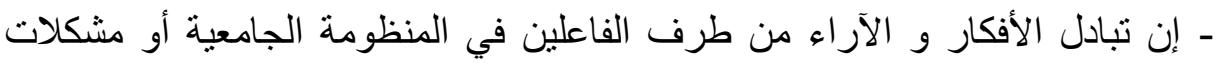

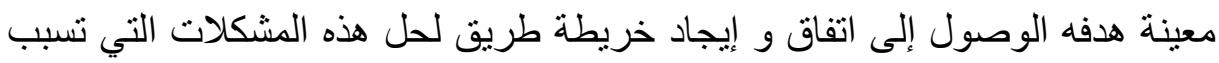

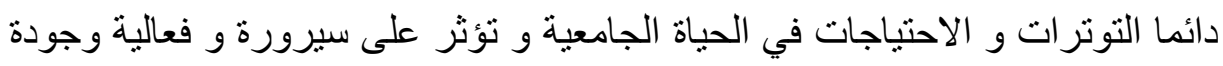
العملية التعليمية في المؤسسة الجامعية و الحكم على نجاح العملية مرتبطة بتحقيق

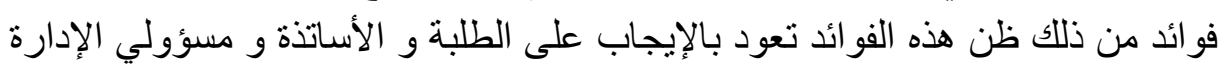

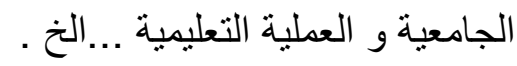

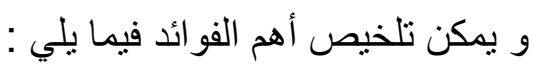

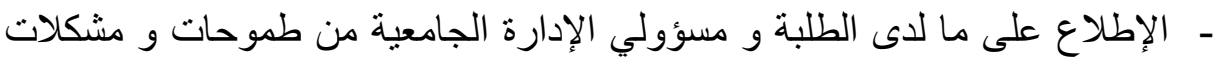

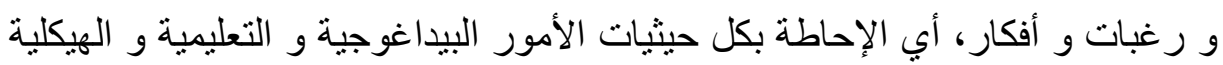

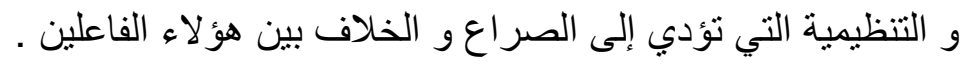

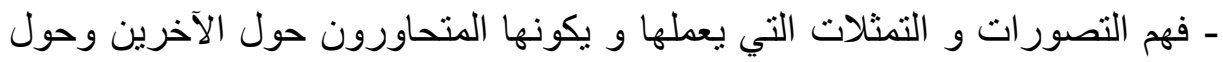

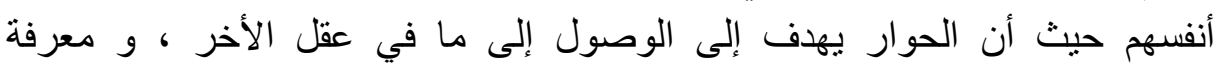

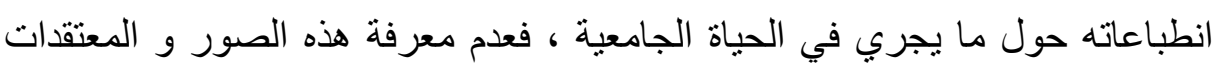

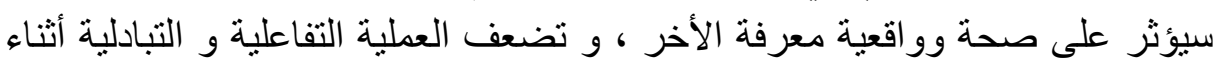

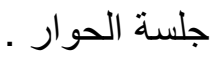

- حفظ الطلبة و خاصة ممثليهم من كل السلوكيات و الممارسات الغير مسؤولة و الغير مقبولة بيداغوجيا داخل الحرم الجامعي ، و التي قد تؤثر على مسلى مسار هم التعليمي

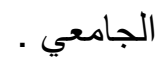
-حل المشكلات و النقائص التي تتخبط فيها المؤسسة الجامعية بإثراك الطلبة و

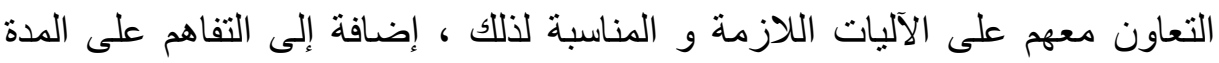
الزمنية لذلك ، و الأولويات المستعجلة و الصبر على الصى المطالب الصعبة التحقيق .

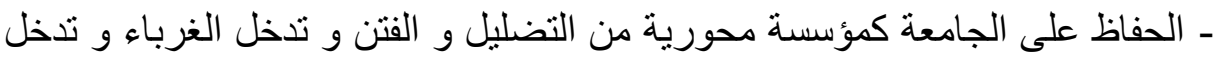
السياسيين باعتبار ها هامة ، و الحفاظ على سيرورة وجودة العملية التعليمية داخلها

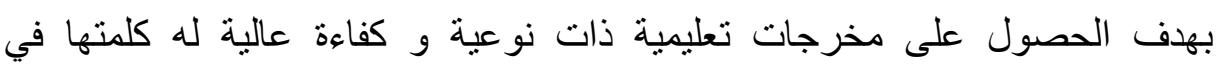

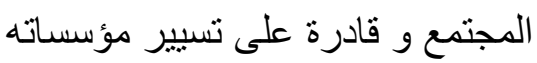

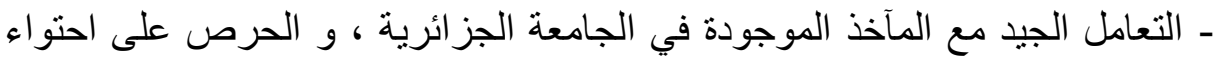

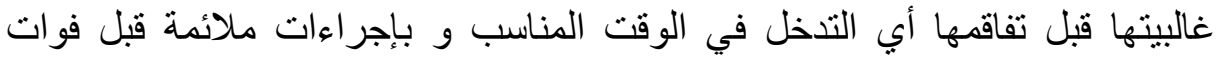

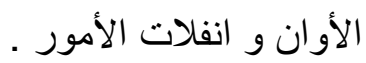

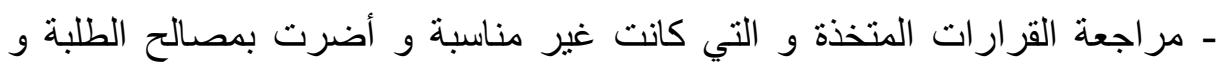

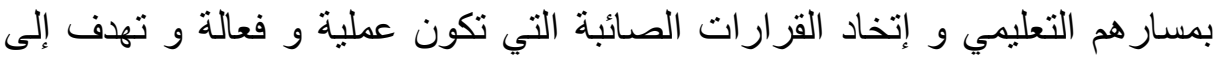

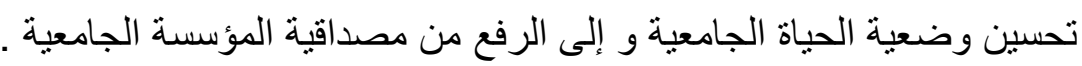

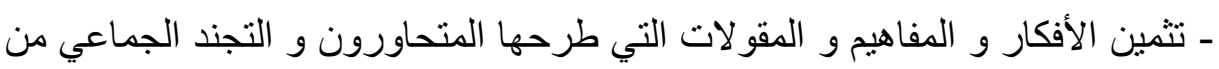

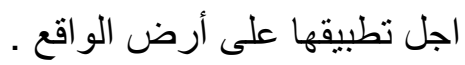
ـ الحد من التوترات و الاستفزازات و الملاسنات اليومية الموجودة في علاقات هؤلاء 
الفاعلين في المنظومة الجامعية ، و العمل على تصغيرها ووضعها في زاوية ضعيفة

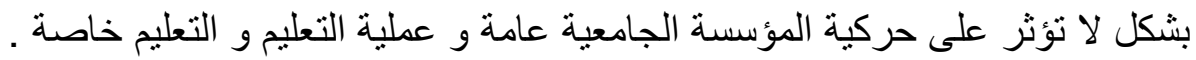

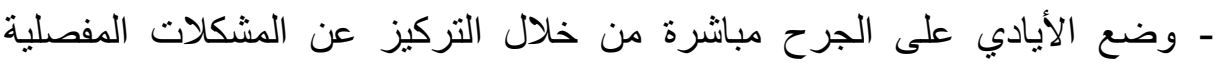
الموجودة داخل الوسط الجامعي و تحديد المسؤوليات حتى يتم الاستعجال في حلها و

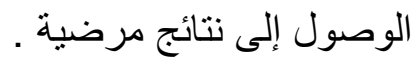

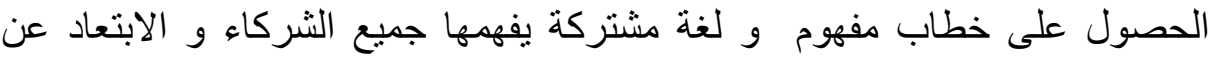
منطق التعالي و الدونية في التعامل مع هذه المطبات الحاصلة في الوسط الجامعي .

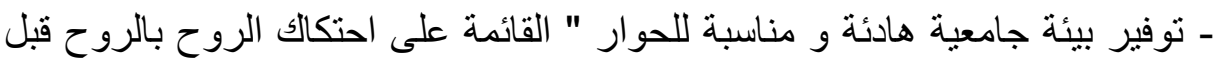

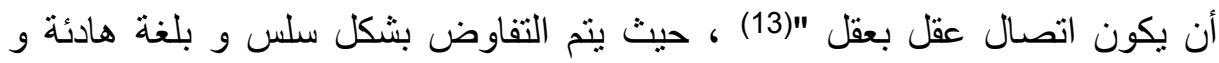

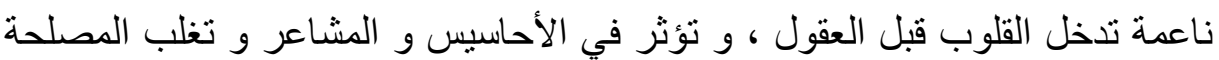

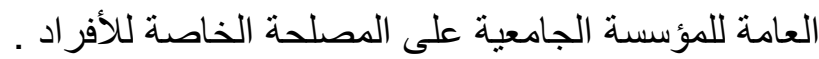

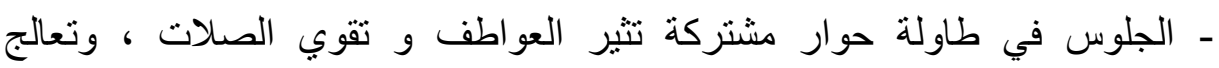

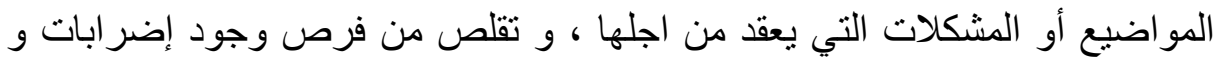
احتجاجات طلابية في المرحلة القادمة .

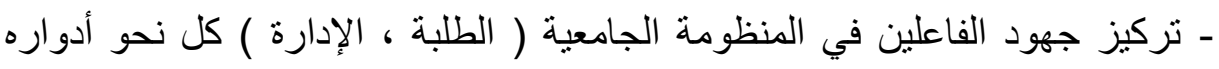

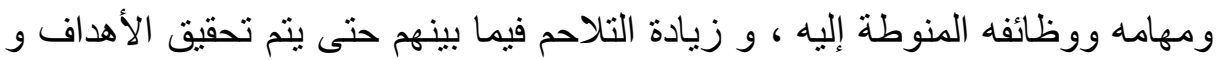

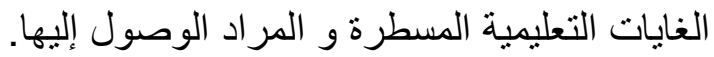

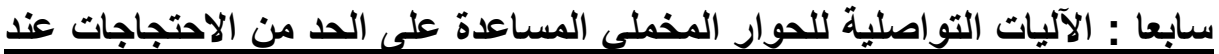
الطلبة: :

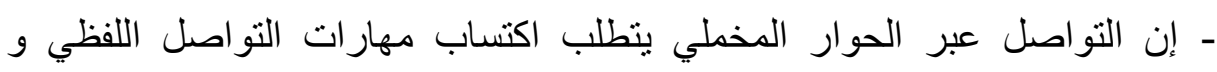

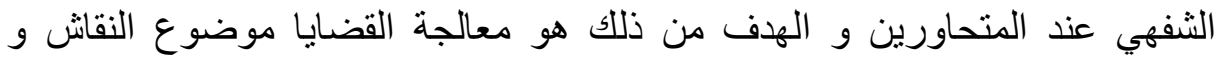
الخلاف معالجة جادة و عقلية و موضوعية وهذا النوع من الحوار هو فن و وله علم قائم

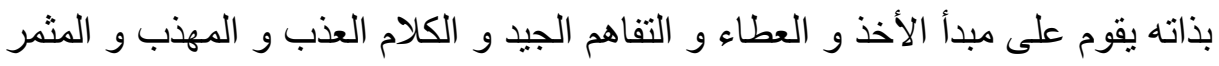
...الخ و يمكن إيجاد الآليات المساعدة على حل مشاكل الطلبة و الحد من إضراباتهاتهم

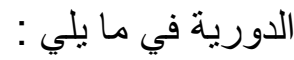
ـ الحرص على فهم مشاعر المحاورين من خلال تحريك عو اطفهم بالكلام المعسول

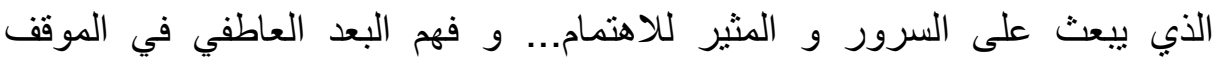

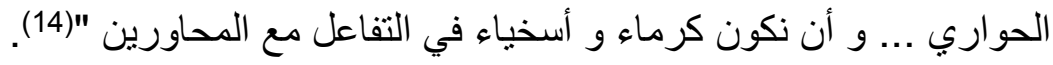

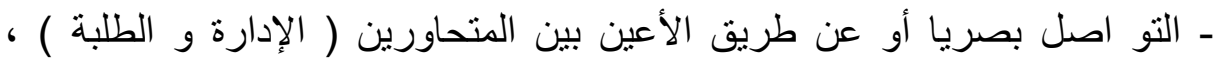

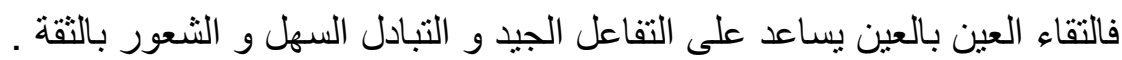

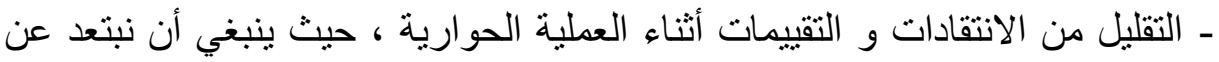

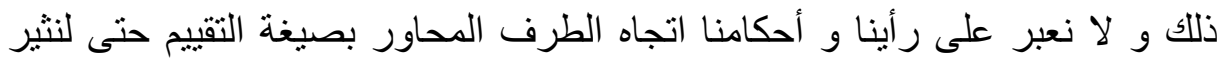
حفيظة الطرف الأخر المحاور و لا نجرح مشاعر الغضب و تقلب الأمور إلى توترات

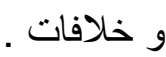
ـالتقليل في المواعظ و النصائح و التوجيهات لأنها تنقص من مشاعر الطرف المحاور و تشعره بالنص و الدونية و عدم النضج و تجدد الجروح بدل تضميدها.

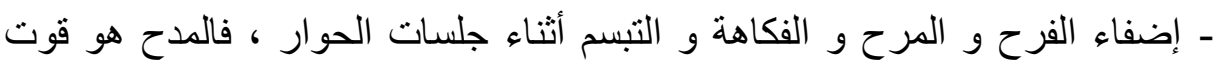


الروح وقد دلت بعض الدراسات على هرمون (الدوبامين ) الذي يفرزه الجسم عند

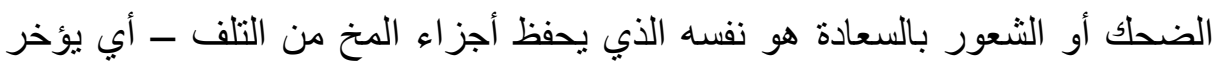

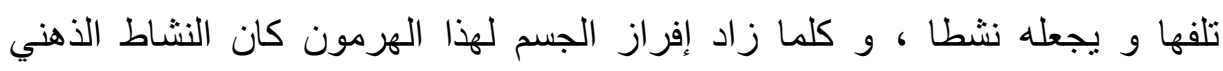

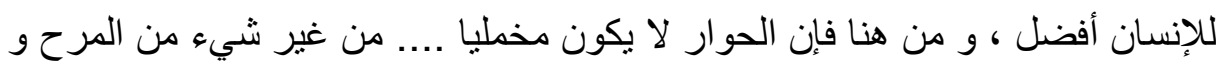

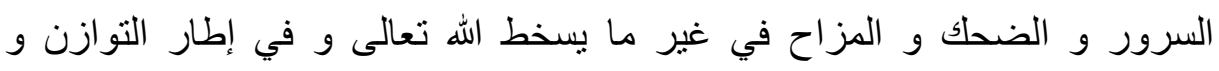

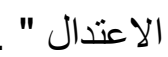

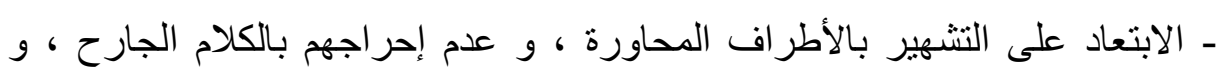

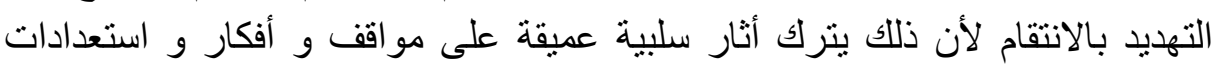

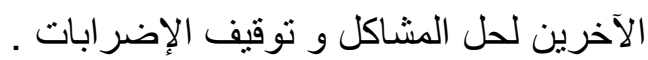

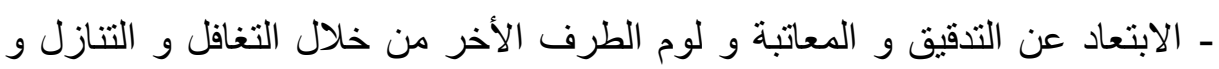
الإعر اض عن أشياء و عدم التركيز على تفاصيل جزئية و الذهاب مباشرة لأشئ نحو الأمور الخلافية و محاولة در استها. ـ أناقة اللسان هي ترجمة لأناقة الروح ، ، رالذين يستخدمون تعبيرات خشنة يحملون

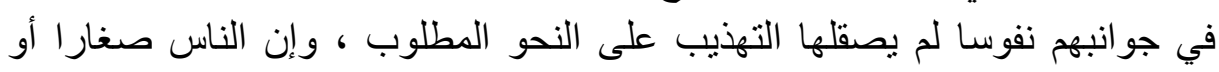

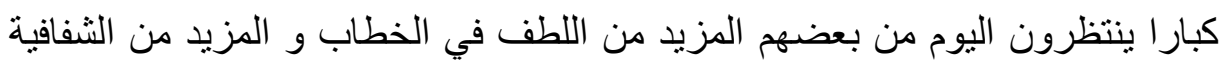

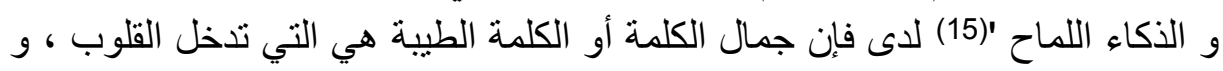

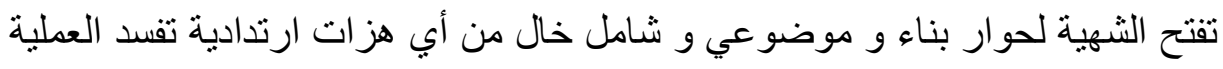

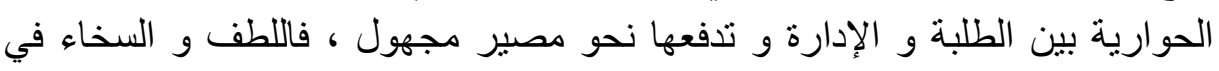

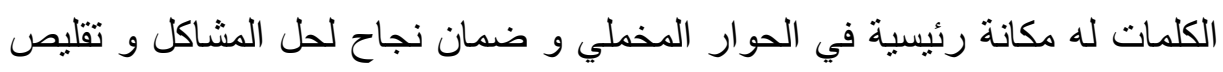

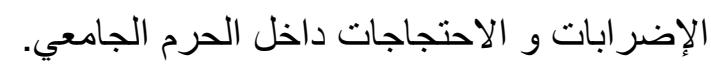

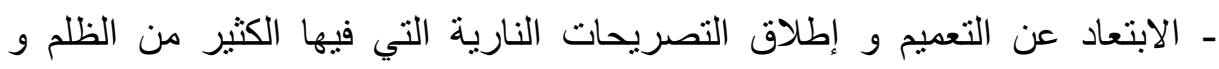

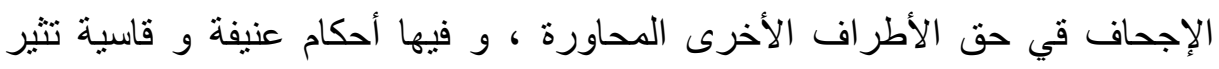

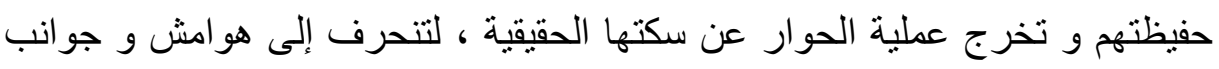

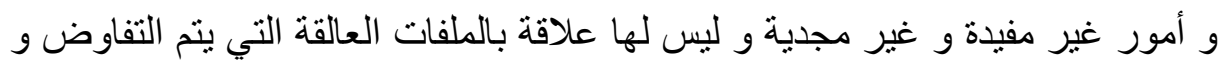

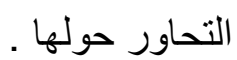
ـ القدرة على التفكير فيما يقال بالسرعة المعقولة مع الاستعداد لقبول الأخر و طرحه

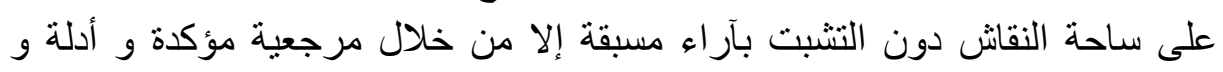

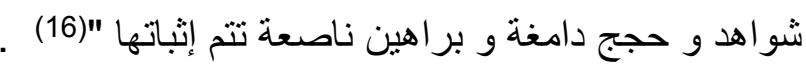

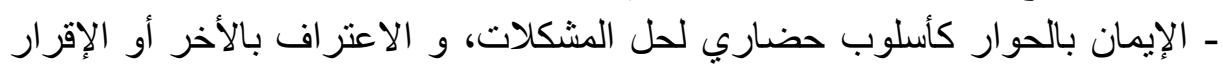

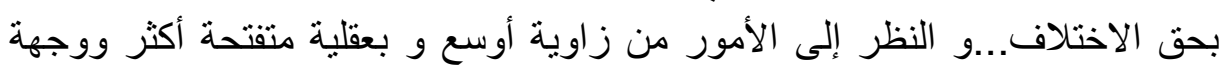
نظر أغنى و أعمق.... و تتمية ثقافة النقد للذات و مراجعة النفس الأس ، و و أن نعتبر الرأي

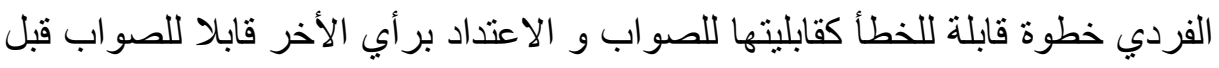

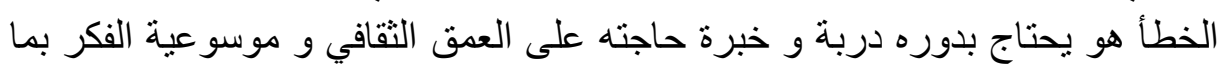

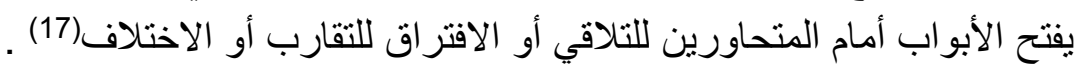

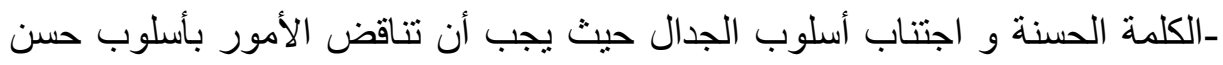

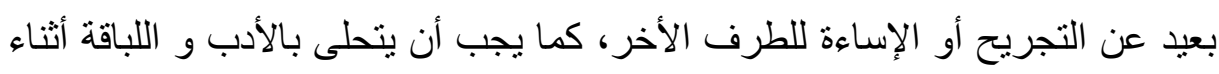




$$
\text { الحديث و أن ييتعد على السخرية و إثارة غضب الطرف الأخر. }
$$

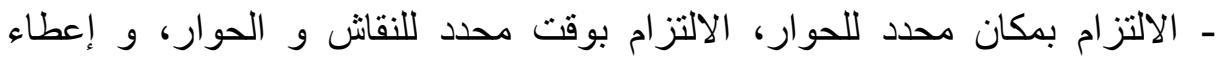

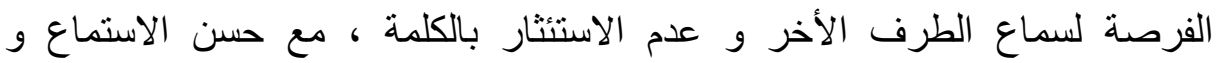

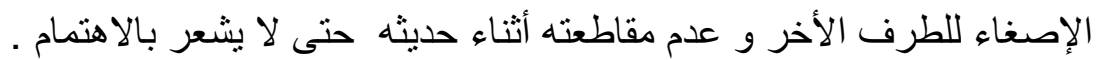

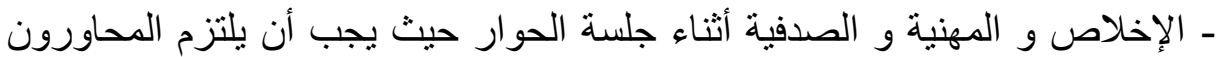

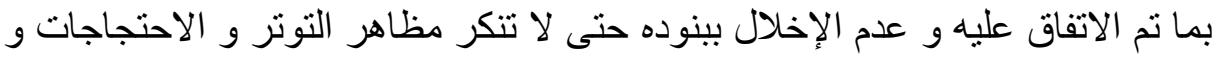

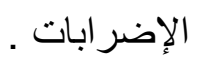

- طرح مباثر للأمور العالقة و عدم تغيير الموضوع المر اد در استه مع عدم الإصرار

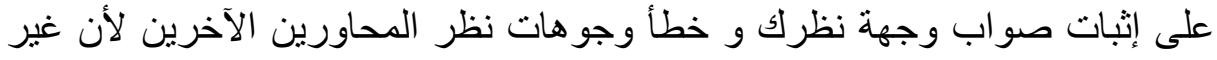

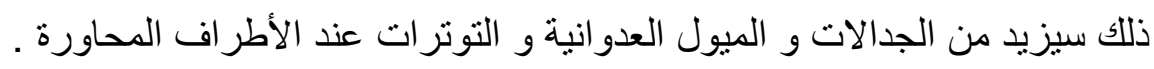

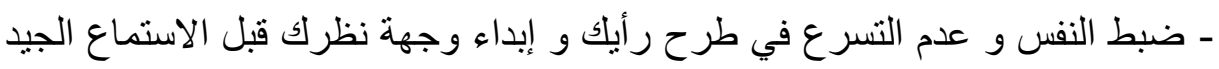

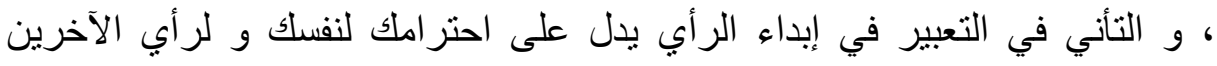

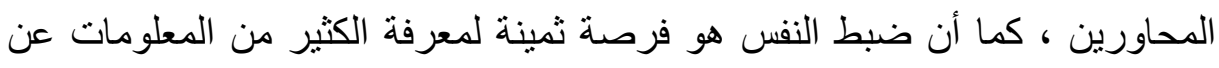

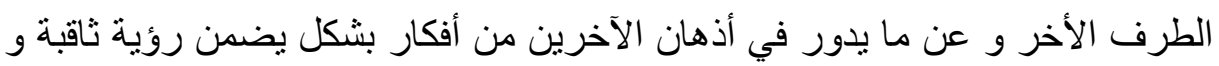

صحيحة للحلول المناسبة .

الخاتمة :

إن العديد من المسؤولين في عدة مؤسسات و منها الجامعة دائما ما يضعون أنفسهح

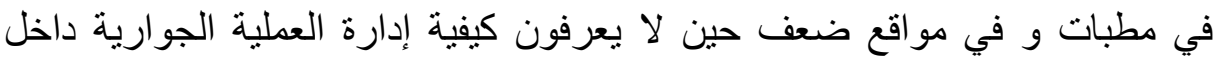

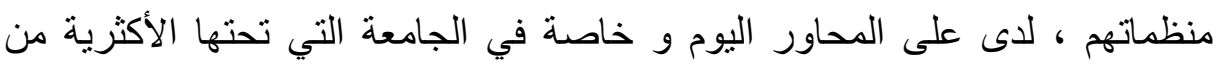

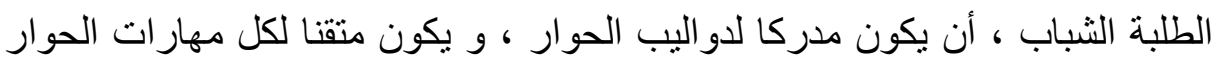

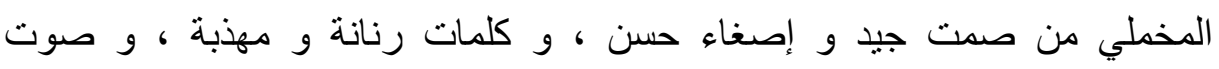

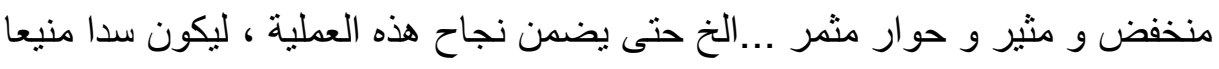

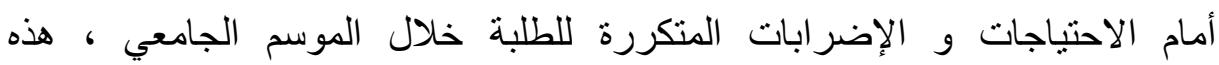

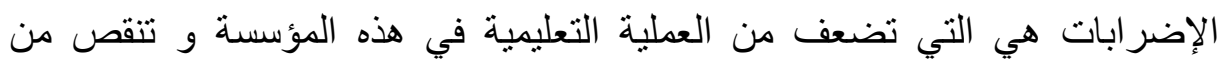

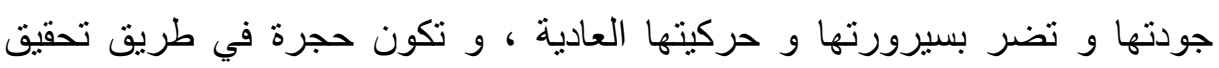
الأهداف التعليمية.

الالتفاعل الجيد و التواصل الحسن بين المتحاورين هو السبيل لتقوية الروابط

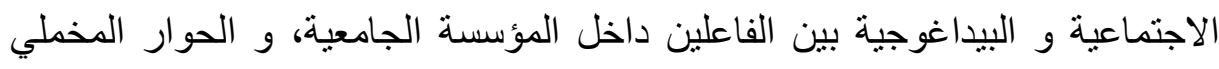

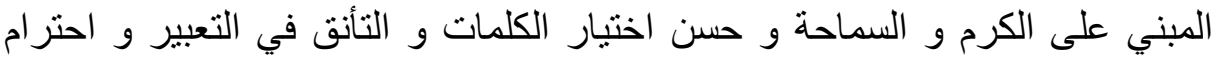

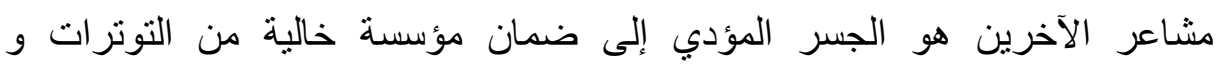
الإضر ابات و محققة لمصالحها و أهدافها العامة و مصدالح و أهداف الأفر اد المنتمين 


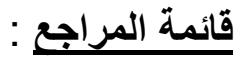

1- ابن منظور ، (1993) ، تهذيب لسان العرب ، ج1،ط1، لبنان ، بيروت : دار

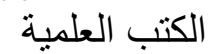
2- ابن منظور ، ( 1997) ، لسان العرب ، المجلد 2 ، ط1 ، لبنان ، بيروت : دار

3 - الرفاعي منصور عبيد ، ( 2004) ، الحوار و آدابه و أهدافه ، ط1 ، مصر : مركز الكتاب للنشر 4- بكار ، عبد الكريم ، (2011) ، التراب ، التواصل الأسري : كيف نحمي أسرنا من التفكك

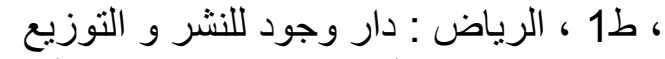

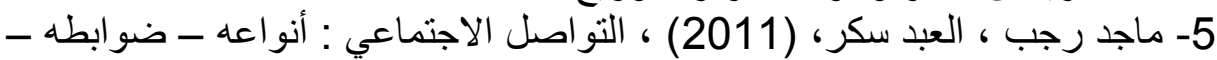

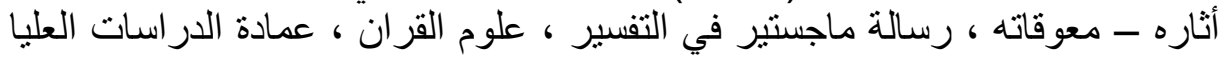

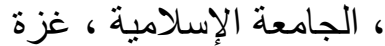
6- المعلم بطرس السناني ، محيط المحيط ، (1993 ) ، قاموس مطول للغة العربية ،

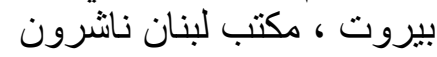

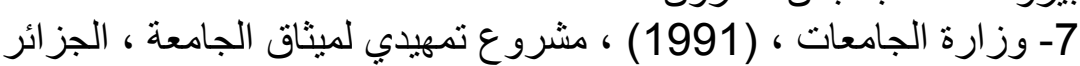

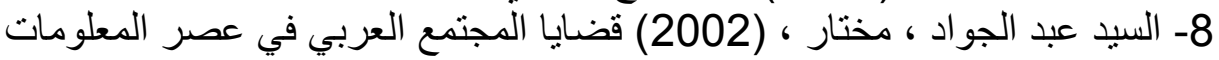
، المؤتمر السنوي العاثر ، القاهرة : دار الفيد الفكر العربي .

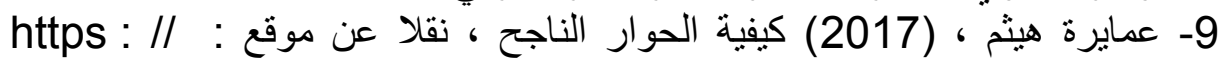
mawdoo3.com (retrievied,28.02.2019) 10- التطاوي عبد الله ، (2006 ) ) ، الحوار الثقافي : مشروع التواصل و الانتماء ،

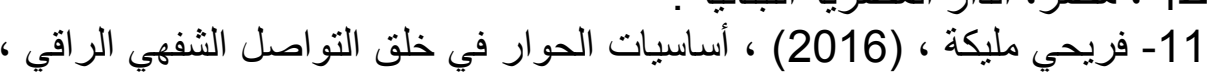

https M //www.oudnad.netsspip (retrieved,28.02.2019)

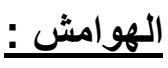

(1)- بكار ، عبد الكريم ، (2011)، التواصل الأسري : كيف نحمي أسرنا من التفكك ، ط1، الرياض:

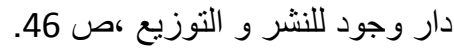


(2)- ابن منظور ، (1993) ، تهذيب لسان العرب ، ج1،ط1، لبنان ، بيروت : دار الكتب العلمية

(3)-ابن منظور ، (1997) ، لسان العرب ، المجلد 2 ، ط1 ، لبنان ، بيروت : دار صادر ص 182

(4)-الرفاعي منصور عبيد ، ( 2011) ، الحوار و آدابه و أهدافه ، ط1 ، مصر : مركز الكتاب

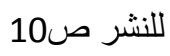

$$
\text { (5)-بكار ، عبد الكريم ، (2011) ، مرجع سابق ، ص } 10 \text { (450) }
$$

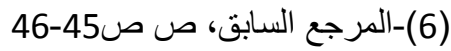

$$
\begin{aligned}
& \text { (7)- المرجع السابق ص ص ص } 46 \text { - } 55 \text { (6) }
\end{aligned}
$$

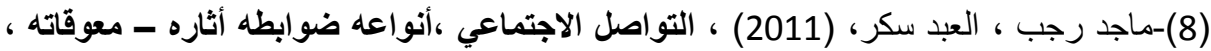

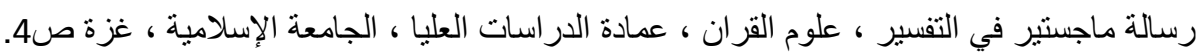

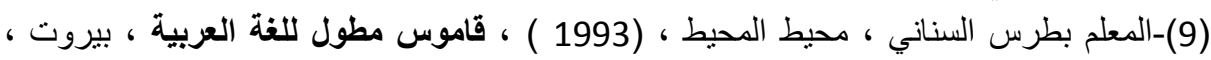

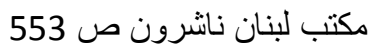

(10)-وزارة الجامعات ، (1991) ، مشروع تمهيدي لميثاق الجامعة ، الجزائر ص111.

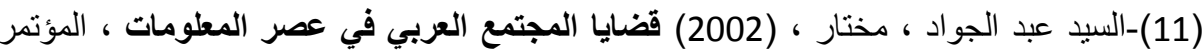

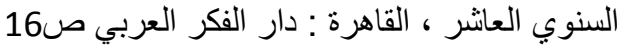

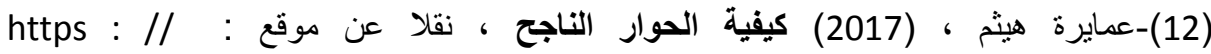

(mawdoo3.com (retrievied,28.02.2019

$$
\begin{aligned}
& \text { (13)- بكار عبد الكريم ، مرجع سابق، ص2929 }
\end{aligned}
$$

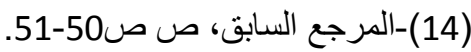

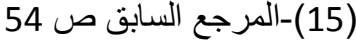

(16)-فريحي مليكة ، (2016) ، أساسيات الحوار في خلق التواصل الثفهي الراقي ، نقلا

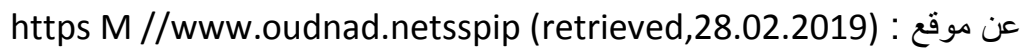

(17)-التطاوي عبد الله ، (2006 ) ، الحوار الثقافي : مشروع التو اصل و الانتماء ، ط1 ، مصر،

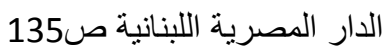

